\title{
Minireview Role of survivin and its splice variants in tumorigenesis
}

\author{
$\mathbf{F} \mathbf{L i}^{*, \mathbf{I}}$ \\ 'Department of Pharmacology and Therapeutics, Grace Cancer Drug Center, Roswell Park Cancer Institute, Elm and Carlton Streets, Buffalo, NY I4263, \\ USA
}

Survivin, a unique member of the inhibitor of apoptosis (IAP) protein family, is highly expressed in cancer but is undetectable in nonproliferating normal adult tissues, suggesting a potential role in tumorigenesis. Differential splicing of survivin pre-mRNA results in three new survivin variants, survivin- $\Delta$ Ex3, survivin-2B, and survivin-3B. Loss of survivin-2B expression was found in the later stage of cancer development, while survivin and survivin- $\Delta \mathrm{E} \times 3$ are not, suggesting a differential role of them in tumour development. In this minireview, the author intends to summarise and discuss the current data relevant to the role of survivin and its splicing variants in tumorigenesis, which may facilitate further investigation in this interesting area.

British Journal of Cancer (2005) 92, 212-2 I6. doi: I 0.1038/sj.bjc.6602340 www.bjcancer.com

Published online 21 December 2004

(C) 2005 Cancer Research UK

Keywords: survivin; survivin-2B; survivin- $\Delta$ Ex3; tumorigenesis

\begin{abstract}
Abnormal inhibition of physiological apoptosis during homeostasis is thought to be a critical step for carcinogenesis or tumorigenesis, although the underlying mechanism is not fully understood. In addition to the Bcl-2 protein family, which includes both antiapoptotic and proapoptotic factors, the inhibitor of apoptosis (IAP), a relative new protein family, is mainly involved in antiapoptosis. This family of proteins are characterised by a signature domain of about 70 amino acids (aa) termed baculovirus IAP repeat (BIR), which is evolutionarily conserved including virus, yeast, nematode, fly, and mammalian. Survivin, a unique member in the IAP family, is undetectable in most normal adult tissues but is highly expressed in cancer. Survivin is cell cycle regulated, and involved in both control of programmed cell death (apoptosis) and regulation of cell division. The molecular mechanism by which survivin counters against apoptosis and facilitates cell division has been extensively explored in cancer cells (Li, 2003). More effort appears to be required for the delineation of the role of survivin in tumorigenesis at the molecular level. Development of new model systems may be desired to unravel the mechanism for the function of survivin and its variants in tumorigenesis. Nevertheless, there is ample data indicating that survivin and its variants may have a differential role in tumorigenesis and cancer development. This minireview will summarise the data as a collective reference, with the intent to indicate where the current research has brought us and to help in the elucidation of future research directions in this interesting area.
\end{abstract}

\section{SURVIVIN IN TUMORIGENESIS}

The initial indication for survivin to be involved in carcinogenesis is the observation that survivin is highly expressed in cancer as

*Correspondence: Dr F Li; E-mail: fengzhi.li@roswellpark.org

Received 30 September 2004; revised 9 November 2004; accepted I5 November 2004; published online 21 December 2004 well as during development, but it is undetectable in nonproliferating adult tissues. Later studies indicated that survivin expression also occurs in very early stages of cancer, and there is a direct correlation of survivin expression with the stage of cancer and other malignant parameters. Selected studies with emphasis on tumorigenesis are discussed below.

\section{Role of survivin in colorectal tumorigenesis}

Kawasaki et al (1998) reported that survivin expression reduced apoptotic index in both bcl-2-positive and -negative colorectal carcinomas, and patients with low apoptotic index in their tumours had significantly worse survival rates. This observation suggests that survivin is an independent prognostic factor. Consistently, immunohistochemical analysis of 52 colorectal carcinoma samples indicated that apoptotic index is significantly lower but PCNA labelling index is significantly higher in the 27 survivin-positive cases than in the 25 survivin-negative cases (Chen et al, 2004). This suggests that survivin expression not only inhibits apoptosis but it also is associated with cell proliferation.

Reports from Kawasaki et al (2001) and Lin et al (2003) further explored the role of survivin in colorectal carcinogenesis. Immunohistochemical analysis of 143 hyperplastic polyps, 171 adenomas with low dysplasia, 42 adenomas with high dysplasia, and 60 carcinomas indicated that the immunoreactivity of survivin (but not bcl-2) significantly increases in the transition from adenoma with low dysplasia to high dysplasia/carcinoma. This transition is significantly associated with a decrease of apoptotic index, and an increase of Ki-67 labelling index and microvessel density (Kawasaki et al, 2001). Lin et al (2003) showed that the positivity of survivin significantly increased in the transition from normal mucosas $(0.0 \%)$ to adenomas with low-grade dysplasia $(31.7 \%)$ to high-grade dysplasia $(56.7 \%)$ or carcinomas $(63.2 \%)$ although survivin expression was not associated with the histological differentiation grade of colorectal carcinoma. Together, these results indicate an important role of survivin in 
colorectal tumorigenesis, which was further supported by the study of signalling pathways relevant to colorectal tumorigenesis in other reports (see below).

Using HT-29 cell lines with inducible wild-type APC or dominant-negative TCF-4, Zhang et al (2001) showed that wtAPC downregulates survivin expression via suppressing $\beta$-catenin/ TCF-4 signalling. In addition, survivin expression is preferentially in the lower crypt and inversely correlates with the expression level of wild-type APC. Consistent with the finding from Zhang et al, Kim et al (2003) reported that $\beta$-catenin/TCF signalling in HCT116 cells increases survivin promoter activity about 6-12-folds in comparison with that in $\mathrm{HeLa}$ cells lacking such signalling. Mutagenesis of the two proximal TCF-binding elements abolished survivin promoter activity by $75-79 \%$. Survivin expression is strong in embryonic intestinal crypts, but lost in TCF-4 knockout intestinal crypts (Kim et al, 2003). Expression of nondestructible $\beta$-catenin mutants increases survivin expression and protected against UV-B-induced apoptosis (Kim et al, 2003). Treatment of HT-29 colon carcinoma cells with the chemopreventive drug sulindac induces $\beta$-catenin degradation and decreases the expression of survivin but not Bcl-2 (Zhang et al, 2004b).

On the basis of these findings, one mechanism for colorectal tumorigenesis appears to be that APC gene mutation results in upregulation of survivin transcription through activation of $\beta$-catenin/TCF-4 signalling. Survivin upregulation increases apoptosis inhibition and cell survival/overgrowth, and initiates tumorigenesis. However, in normal colorectal intestinal crypts, wild-type APC signalling suppresses survivin transcription through inactivation of $\beta$-catenin/TCF-4 signalling. This will increase apoptosis from crypt bottom to top, decrease cell population and block tumorigenesis. Interestingly, it was recently reported that inhibition of Wnt-2 signalling, by either a novel monoclonal antibody against human Wnt-2 ligand or Wnt-2 small interfering RNA, downregulates $\beta$-catenin and survivin, and induces apoptosis in Wnt-2-overexpressing human melanoma (You et al, 2004a) and non-small-cell lung cancer cells (You et al, $2004 \mathrm{~b}$ ), but not in normal cells lacking Wnt-2 expression. In addition, it was also reported that proteasome inhibitor, MG132induced apoptosis is associated with fragmentation of $\beta$-catenin, reduction of TCF transcriptional activity and downregulation of survivin in hepatocellular carcinoma cells (Cervello et al, 2004). These findings suggest that the $\beta$-catenin/TCF-4/survivin pathway may not only be responsible for colorectal tumorigenesis but it may also possibly be responsible for tumorigenesis in other types of cancer.

\section{Role of survivin in virus infection-induced carcinogenesis}

The clue for survivin involvement of virus-induced tumorigenesis comes from the observation that expression of survivin transcript was found in all the nine human T-cell leukaemia virus type I (HTLV-I)-positive T-cell lines examined and downregulation of survivin by antisense oligonucleotides inhibited growth in the HTLV-I-infected MT-1 T-cell line (Mori et al, 2001). Consistently, resveratrol, a chemopreventive agent commonly found in human diet, downregulates survivin expression and induces growth inhibition and apoptosis in HTLV-1-infected cell lines (Hayashibara et al, 2002). In addition, high survivin expression was detected in primary cells of patients with acute adult T-cell leukaemia (ATL), but not in the cells of patients with chronic ATL or normal peripheral blood mononuclear cells (Mori et al, 2001). It will be interesting to know whether this is associated with a difference of HTLV infection in these two types of ATL.

Immunohistochemical analysis of 73 cervical squamous tissues for survivin expression, including 31 normal, 17 low- and 15 highgrade squamous intraepithelial lesions (LSIL, HSIL), and 10 squamous cell carcinomas (SCC) from cone biopsy and hysterectomy specimens, indicated that although nuclear staining of survivin was detected in normal mucosa, LSIL, and HSIL, survivin expression is highest in human papillomavirus (HPV)-infected tissue samples. Moreover, in situ hybridisation of serial sections showed the colocalisation of HPV and survivin (Frost et al, 2002). This report indicated that the histologic correlation between nuclear staining of survivin and HPV infection suggests involvement of survivin in HPV-mediated disruption of normal cellular maturation (Frost et al, 2002). However, alternative roles of survivin upregulation in HPV-infected cells may exist (see below). In another report, immunohistochemical analysis for survivin expression and nested PCR plus direct sequencing for detection of HPV presence showed that survivin expression was evident in $57.1 \%$ (four out of seven) $\mathrm{HPV}^{+}$ (high level) and $100 \%$ (four out of four) $\mathrm{HPV}^{-}$(low level) oral SCC, and $85.7 \%$ (six out of seven) $\mathrm{HPV}^{+}$and $55.5 \%$ (five out of nine) $\mathrm{HPV}^{-}$oral leucoplakias, and $0 \%$ (zero out of 20) control oral mucosa samples, and that survivin expression was found to be greater in $\mathrm{HPV}^{+}$precancerous lesions than in $\mathrm{HPV}^{-}$ones (Lo Muzio et al, 2004). Since HPV has been thought to be involved in the development of several oral diseases, such as premalignant mucosal lesions and oral carcinoma, these authors suggested that survivin may directly or indirectly be regulated by HPV and may be involved in HPV-induced deregulation during maturation of squamous epithelium through modulation of the apoptotic processes (Lo Muzio et al, 2004). Consistent with the possibility that HPV may induce survivin expression, cDNA microarray indicated that HPV E6/E7 upregulates survivin (Nees et al, 2000). However, Veress et al found the HPV E6 protein can transcriptionally upregulate survivin promoter activity over 10 -folds in HeLa cells in comparison with empty vector control, while HPV E7 alone has no effect on survivin transcription (personal communication, University of Debrecen, Hungary). An interesting question is whether upregulation of survivin after virus infection is a universal phenomenon. Zhao et al reported that adenovirus type-2 also upregulates survivin in HeLa cells after infection (Zhao et al, 2003). An RNA probe derived from the adenovirus type 2-infected HeLa cells was used to examine cDNA microarrays of 12309 unique genes. The results showed that survivin is one of the top 53 genes with significant changes (35 up and 18 down) and significantly upregulated (Zhao et al, 2003). Consistent with this finding, Punga and Akusjarvi (2003) showed that the adenovirus $2 \mathrm{E} 1 \mathrm{~B}-55 \mathrm{~K}$ protein upregulates survivin by binding to and blocking p53 as a transcriptional repressor on survivin promoter.

The notion that upregulation of survivin during virus infection may have a role in facilitating either virus production or virusinduced tumorigenesis comes from two additional recent reports (Marusawa et al, 2003; Zhu et al, 2003). Zhu et al showed that human immunodeficiency virus type 1 (HIV-1) transcriptionally upregulates survivin expression, that Vpr, an HIV-1 accessory protein, is necessary and sufficient for this effect, and that survivin may be actively involved in regulating cell viability during HIV-1 infection (Zhu et al, 2003). This likely facilitates virus production by increasing host cell viability. More interestingly, Marusawa et al showed that survivin forms complexes with a cellular protein, hepatitis B X-interacting protein (HBXIP), which was originally recognised for its association with the $\mathrm{X}$ protein (HBX) of hepatitis $B$ virus (HBV). Survivin-HBXIP complexes, but neither survivin nor HBXIP individually, bind procaspase-9, preventing its recruitment to Apaf1, and thereby selectively suppressing apoptosis initiated through the mitochondria/cytochrome $c$ pathway (Marusawa et al, 2003). Moreover, viral HBX protein also interacts with the surviving-HBXIP complex and suppresses caspase activation only in the presence of survivin (Marusawa et al, 2003). These observations highlighted that survivin is critical for HBV-induced apoptosis inhibition. Thus, this study provides direct evidence that survivin is likely involved in HBV pathogeninduced hepatocellular tumorigenesis. 


\section{Additional evidence from selected reports}

Lo Muzio et al (2003) showed that survivin is immunohistochemically present in 10 out of 30 cases $(33 \%)$ of oral precancerous lesions without malignant progression, but in 15 out of 16 cases (94\%) of oral precancerous lesions which evolved into full-blown SCC, and that survivin positivity appears to be an early event and a critical factor for tumor progression from precancerous lesion. Zhang et al showed that the immunohistochemical reactivity of survivin is $4.2 \%$ (four out of 96 ) in normal mammary, $5.4 \%$ (three out of 56) in cystic hyperplasia mammary, $42.7 \%$ (five out of 12) in atypical hyperplasia mammary, and $72.3 \%$ (86 out of 119 ) in breast carcinoma, and that the positive rates in the last two groups are statistically significant in comparison with the former two groups (Zhang et al, 2004a). Kim et al (2002) reported that in 41 cervical intraepithelial neoplasia (CIN) and SCC tissues, survivin expression is $66.7 \%$ in low-grade (CIN I), $87.5 \%$ in high-grade (CIN II and III), and $100 \%$ in SCC, which is statistically significant. These observations provide additional evidence for the involvement of survivin expression in tumorigenesis.

However, the most interesting observation comes from a study of the skin carcinogenesis using a survivin-transgenic (Tg) mouse model (Allen et al, 2003). Early observation from Grossman et al (1999)indicated that survivin is immunohistochemically expressed in 17 of $21(81 \%)$ of basal cell carcinomas of both nodular and morpheaform subtypes, and in 24 of 26 (92\%) of cutaneous SCC. Survivin is also expressed in 19 premalignant lesions of Bowen's disease and hypertrophic actinic keratosis. This suggests that survivin expression occurs early during keratinocyte transformation and may play a role in carcinogenesis. Paradoxically, Allen et al (2003) used DMBA carcinogen and PMA carcinogenic promoter to induce skin tumour formation in a $\mathrm{Tg}$ mouse model in which survivin is specifically overexpressed in skin driven by the keratin-14 gene promoter. These authors found that tumour formation was less frequent ( 31 vs $43 \%$ ) and significantly delayed in $\mathrm{Tg}$ mice in comparison with non- $\mathrm{Tg}$ littermates. However, papilloma regression was not observed in Tg mice, whereas $20 \%$ of papillomas regressed in non-Tgs. One SCC was generated in $\mathrm{Tg}$ mice, whereas none were seen in non-Tgs. In another set of experiments, Tg mice with p53+I- background were used. Again, DMBA/PMA-induced tumour formation was less (71 vs 89\%) and significantly delayed in $\mathrm{Tg}$ p $53+/-$ animals in comparison with p53 + I- non-Tgs. However, papilloma regression was also not observed in $\mathrm{Tg} \mathrm{p} 53+1-$ mice, whereas $10 \%$ of papillomas regressed in $\mathrm{p} 53+1-$ non-Tgs. The rate of papilloma progression to SCC was $21 \%$ in $\mathrm{Tg}$ p $53+I-$ mice in comparison with $12 \%$ in p53+I- non-Tgs (Allen et al, 2003). Together, these authors reported interesting results. The question is how to explain the negative effect of survivin on skin carcinogenesis and the positive effect on tumour progression. A recent report from the same research group showed that apoptosis inhibition by Tg expression of survivin enhances mutant clone creation and malignant progression during UV-B treatments in the skin carcinogenesis model, but inhibition of apoptosis by survivin in the neighbour survivin-Tg-transgenic skin cells restricts mutant clone expansion (tumour formation) (Zhang et al, 2004c). This may provide an explanation for the paradoxical results above. Anyway, the model will be significantly strengthened, if it could gain an insight into a molecular basis to explain why apoptosis in the neighbour normal cells is so important for the mutant clone to be expanded (tumour growth) in skin.

Alternatively, in my personal view, the results from Allen et al may also suggest that artificial overexpression of survivin in normal tissues may show a different role from survivin expression in abnormal precancerous and cancerous tissues. For example, the artificial overexpression of survivin in normal mouse skin may counter against the process of DMBA/PMA-induced carcinogenesis. However, through a selection process driven by DMBA/PMA treatments, individual skin cells with additional genetic and/or biochemical abnormalities will give survivin a different background for function. In the latter environment, survivin plays a positive role in tumour growth/progression for promoting papilloma regression and promoting conversion to SCC.

\section{SURVIVIN VARIANTS IN TUMORIGENESIS}

Human survivin gene has four dominant $(1,2,3$, and 4) and two hidden ( $2 \mathrm{~B}$ and $3 \mathrm{~B}$ ) exons. Alternative splicing of its pre-mRNA produces four different mRNAs, which encode four distinct proteins, survivin, survivin-2B, survivin- $\Delta \mathrm{Ex} 3$ (Mahotka et al, 1999), and survivin-3B (Badran et al, 2004). Survivin (142 aa) is derived from exons $1-4$; survivin-2B (167 aa) has an additional 23 aa derived from a 69-bp cryptic exon (2B) within intron 2 , which is spliced into survivin mRNA in frame between exons 2 and 3; survivin- $\Delta \mathrm{Ex} 3(137 \mathrm{aa})$ is derived from exons 1,2 , and 4 , a frameshift read-through variant due to exon 3 escape; and the recently reported survivin-3B (120 aa) consists of the N-terminal 113 aa of survivin (coded by exons 1, 2, and 3) plus seven new aa sequences at C-terminal tail encoded by a DNA sequence (exon 3B) from intron 3 of survivin. Currently, there is no strong evidence suggesting that survivin splicing variants are involved in tumorigenesis. However, Islam et al (2000) reported that the expression of survivin-2B is predominant in some neuroblastoma with a good prognosis, but it is expressed at low levels in most malignant tissues. In renal cell carcinomas (RCC), while the expression levels of survivin and survivin- $\Delta \mathrm{Ex} 3$ did not show a decrease in late tumour stages in comparison with the early and intermediate tumour stages in 57 clinical RCC samples, survivin2B expression was significantly decreased in late tumour stages (Mahotka et al, 2002a), indicating a possible unfavourable role of survivin-2B in cancer development. In gastric cancer, the expression of survivin- $\Delta \mathrm{Ex} 3$, survivin-2B, and survivin (dominant transcript) was found in cancer tissues, irrespective of their histological type, grade, or stage (Krieg et al, 2002) (Meng et al, 2004). However, survivin-2B expression was significantly decreased in later tumour stage (III + IV) in comparison with early stage (I + II) (Krieg et al, 2002; Meng et al, 2004), and inversely correlated with tumour differentiation and invasion (Meng et al, 2004). Interestingly, although Krieg et al (2002) reported that the expression of survivin and survivin- $\Delta \mathrm{Ex} 3$ remained unchanged in different stages of cancer, Meng et al (2004) showed that the expression level of survivin- $\Delta \mathrm{Ex} 3$ is inversely correlated with apoptotic index. It was also reported that survivin-2B expression was dominant in benign brain tumours in comparison with the malignant ones (Yamada et al, 2003), and that survivin- $\Delta \mathrm{Ex} 3$ expression was prominent in comparison with survivin-2B expression in survivin-expressing acute lymphocytic leukaemia (ALL) and chronic lymphocytic leukaemia (CLL) patient bone marrow samples (Nakagawa et al, 2004). Structurally, a novel antiapoptotic protein encoded by open reading frame $\mathrm{K} 7$ of Kaposi's sarcoma-associated herpesvirus bears a resemblance to survivin- $\Delta \mathrm{Ex} 3$ (Wang et al, 2002), further suggesting a possible antiapoptotic role for survivin- $\Delta \mathrm{Ex} 3$. In addition, the different subcellular localisation of survivin- $\Delta \mathrm{Ex} 3$ (in nucleus) and survivin-2B (in cytoplasm) (Mahotka et al, 2002b) also suggests their potential different roles. Collectively, these observations indicated that survivin- $\Delta \mathrm{Ex} 3$ and survivin-2B may play an opposing role in tumour progression and/or tumorigenesis.

Interestingly, in soft tissue sarcoma (STS), 36 of 56 STS (64\%) were survivin transcript-positive, and 15 of the 36 survivinpositive samples were survivin- $\Delta \mathrm{Ex} 3$ transcript-positive, but survivin-2B transcripts were undetectable (Kappler et al, 2001). Similarly, among the 79 gastric tumour samples, the expression of survivin, survivin-2B, and survivin- $\Delta \mathrm{Ex} 3$ was 79 (100\%), 62 (78.5\%), and 51 (64.6\%), respectively (Meng et al, 2004). 
Additionally, studies from Islam et al (2000) indicated that while the expression of survivin was downregulated during retinoic acidinduced apoptosis in CHP134 neuroblastoma cells, survivin-2B expression was unchanged or slightly increased during apoptosis. These observations suggest that the expression of these survivin variants could be differentially regulated. Consistent with this notion, recent studies from Zhu et al (2004) showed that one of the mechanisms for p53 to induce apoptosis in ALL cells is through differential modulation of survivin and its variants. Doxorubicinactivated p53 upregulated survivin-2B but downregulated survivin and survivin- $\triangle \mathrm{Ex} 3$. Therefore, from a cancer prevention and therapeutics point of view, the idea that survivin-2B may act as a natural antagonist against the function of survivin and/or survivin$\Delta \mathrm{Ex} 3$ may lead to novel approaches for cancer prevention and/or therapeutics through differential modulation of the expression of survivin and/or its variants.

\section{SUMMARY REMARKS}

The potential role of survivin in colorectal tumorigenesis appears to be well defined. Many observations also indicate a role of survivin in virus infection-induced carcinogenesis. In addition, studies of normal and different stages of precancerous and cancerous tissues in many other types of cancer also suggest a role of survivin in tumorigenesis. However, the study of DMBA/ PMA-induced carcinogenesis in a mouse skin model (with or without a p53+I- background) that artificially overexpresses survivin in skin using a skin-specific keratin-14 gene promoter, paradoxically revealed that tumour formation was less frequent and significantly delayed in survivin- $\mathrm{Tg}$ mice in comparison

\section{REFERENCES}

Allen SM, Florell SR, Hanks AN, Alexander A, Diedrich MJ, Altieri DC, Grossman D (2003) Survivin expression in mouse skin prevents papilloma regression and promotes chemical-induced tumor progression. Cancer Res 63: $567-572$

Badran A, Yoshida A, Ishikawa K, Goi T, Yamaguchi A, Ueda T, Inuzuka M (2004) Identification of a novel splice variant of the human antiapoptopsis gene survivin. Biochem Biophys Res Commun 314: $902-907$

Cervello M, Giannitrapani L, La Rosa M, Notarbartolo M, Labbozzetta M, Poma P, Montalto G, D'Alessandro N (2004) Induction of apoptosis by the proteasome inhibitor MG132 in human HCC cells: possible correlation with specific caspase-dependent cleavage of beta-catenin and inhibition of beta-catenin-mediated transactivation. Int J Mol Med 13: $741-748$

Chen WC, Liu Q, Fu JX, Kang SY (2004) Expression of survivin and its significance in colorectal cancer. World J Gastroenterol 10: 2886-2889

Frost M, Jarboe EA, Orlicky D, Gianani R, Thompson LC, Enomoto T, Shroyer KR (2002) Immunohistochemical localization of survivin in benign cervical mucosa, cervical dysplasia, and invasive squamous cell carcinoma. Am J Clin Pathol 117: 738-744

Grossman D, McNiff JM, Li F, Altieri DC (1999) Expression of the apoptosis inhibitor, survivin, in nonmelanoma skin cancer and gene targeting in a keratinocyte cell line. Lab Invest 79: $1121-1126$

Hayashibara T, Yamada Y, Nakayama S, Harasawa H, Tsuruda K, Sugahara K, Miyanishi T, Kamihira S, Tomonaga M, Maita T (2002) Resveratrol induces downregulation in survivin expression and apoptosis in HTLV1-infected cell lines: A prospective agent for adult $\mathrm{T}$ cell leukemia chemotherapy. Nutr Cancer 44: 193-201

Islam A, Kageyama H, Hashizume K, Kaneko Y, Nakagawara A (2000) Role of survivin, whose gene is mapped to $17 \mathrm{q} 25$, in human neuroblastoma and identification of a novel dominant-negative isoform, survivin-beta/ 2B. Med Pediatr Oncol 35: 550-553

Kappler M, Kohler T, Kampf C, Diestelkotter P, Wurl P, Schmitz M, Bartel F, Lautenschlager C, Rieber EP, Schmidt H, Bache M, Taubert H, Meye A (2001) Increased survivin transcript levels: an independent negative predictor of survival in soft tissue sarcoma patients. Int J Cancer 95: $360-363$ with non-Tg littermates, although $\mathrm{Tg}$ mice promote papilloma progression into SCC after tumours are initiated from DMBA/PMA treatment. This interesting observation could be due to the apoptosis inhibition by survivin in the neighbour normal cells, which prevents mutant cell expansion (tumour growth), but it may also suggest that the potential role of survivin in normal skin is different from the role in precancerous and cancerous tissues. Therefore, studies of the different role and functional mechanism of survivin between normal and cancerous tissues will help to answer the remaining questions. New research approaches and/or model systems will be required to elucidate the distinct mechanisms by which survivin functions and is regulated in cancer $v s$ normal cells, which will be essential for development of novel and highly effective approaches for cancer prevention and therapeutics. In addition, although data from several reports suggest a potential role of survivin- $2 \mathrm{~B}$ in countering against the function of survivin and/or survivin- $\Delta \mathrm{Ex} 3$ in tumour developments, much more needs to be done before deriving a definitive conclusion.

\section{ACKNOWLEDGEMENTS}

The author thank Ms Paula Pera for editorial assistance and Dr Gyorgy Veress (University of Debrecen, Hungary), who has shared data prior to publication for inclusion in this article. The author apologizes that some relevant reports could not be cited due to space limitation. F $\mathrm{Li}$ is supported by Grants from NIH/NCI (CA109481, USA), Concern Foundation (Beverly Hills, CA, USA), and Elsa U Pardee Foundation (Midland, MI, USA).

Kawasaki H, Altieri DC, Lu CD, Toyoda M, Tenjo T, Tanigawa N (1998) Inhibition of apoptosis by survivin predicts shorter survival rates in colorectal cancer. Cancer Res 58: 5071-5074

Kawasaki H, Toyoda M, Shinohara H, Okuda J, Watanabe I, Yamamoto T, Tanaka K, Tenjo T, Tanigawa N (2001) Expression of survivin correlates with apoptosis, proliferation, and angiogenesis during human colorectal tumorigenesis. Cancer 91: 2026-2032

Kim HS, Shiraki K, Park SH (2002) Expression of survivin in CIN and invasive squamous cell carcinoma of uterine cervix. Anticancer Res 22: $805-808$

Kim PJ, Plescia J, Clevers H, Fearon ER, Altieri DC (2003) Survivin and molecular pathogenesis of colorectal cancer. Lancet 362: 205-209

Krieg A, Mahotka C, Krieg T, Grabsch H, Muller W, Takeno S, Suschek CV, Heydthausen M, Gabbert HE, Gerharz CD (2002) Expression of different survivin variants in gastric carcinomas: first clues to a role of survivin-2B in tumour progression. $\mathrm{Br}$ J Cancer 86: $737-743$

Li F (2003) Survivin study: what is the next wave? J Cell Physiol 197: 8-29

Lin LJ, Zheng CQ, Jin Y, Ma Y, Jiang WG, Ma T (2003) Expression of survivin protein in human colorectal carcinogenesis. World J Gastroenterol 9: $974-977$

Lo Muzio L, Campisi G, Giovannelli L, Ammatuna P, Greco I, Staibano S, Pannone G, De Rosa G, Di Liberto C, D’Angelo M (2004) HPV DNA and survivin expression in epithelial oral carcinogenesis: a relationship? Oral Oncol 40: $736-741$

Lo Muzio L, Pannone G, Leonardi R, Staibano S, Mignogna MD, De Rosa G, Kudo Y, Takata T, Altieri DC (2003) Survivin, a potential early predictor of tumor progression in the oral mucosa. J Dent Res 82: 923-928

Mahotka C, Krieg T, Krieg A, Wenzel M, Suschek CV, Heydthausen M, Gabbert HE, Gerharz CD (2002a) Distinct in vivo expression patterns of survivin splice variants in renal cell carcinomas. Int J Cancer 100: $30-36$

Mahotka C, Liebmann J, Wenzel M, Suschek CV, Schmitt M, Gabbert HE, Gerharz CD (2002b) Differential subcellular localization of functionally divergent survivin splice variants. Cell Death Differ 9: 1334-1342

Mahotka C, Wenzel M, Springer E, Gabbert HE, Gerharz CD (1999) Survivin-deltaEx3 and survivin-2B: two novel splice variants of the 
apoptosis inhibitor survivin with different antiapoptotic properties. Cancer Res 59: 6097-6102

Marusawa H, Matsuzawa S, Welsh K, Zou H, Armstrong R, Tamm I, Reed JC (2003) HBXIP functions as a cofactor of survivin in apoptosis suppression. EMBO J 22: 2729-2740

Meng H, Lu CD, Sun YL, Dai DJ, Lee SW, Tanigawa N (2004) Expression level of wild-type survivin in gastric cancer is an independent predictor of survival. World J Gastroenterol 10: 3245-3250

Mori N, Yamada Y, Hata T, Ikeda S, Yamasaki Y, Tomonaga M, Yamamoto N (2001) Expression of survivin in HTLV-I-infected T-cell lines and primary ATL cells. Biochem Biophys Res Commun 282: 1110-1113

Nakagawa Y, Yamaguchi S, Hasegawa M, Nemoto T, Inoue M, Suzuki K, Hirokawa K, Kitagawa M (2004) Differential expression of survivin in bone marrow cells from patients with acute lymphocytic leukemia and chronic lymphocytic leukemia. Leuk Res 28: 487-494

Nees M, Geoghegan JM, Munson P, Prabhu V, Liu Y, Androphy E, Woodworth CD (2000) Human papillomavirus type 16 E6 and E7 proteins inhibit differentiation-dependent expression of transforming growth factor-beta2 in cervical keratinocytes. Cancer Res 60: $4289-4298$

Punga T, Akusjarvi G (2003) Adenovirus 2 E1B-55K protein relieves p53mediated transcriptional repression of the survivin and MAP4 promoters. FEBS Lett 552: 214-218

Wang HW, Sharp TV, Koumi A, Koentges G, Boshoff C (2002) Characterization of an anti-apoptotic glycoprotein encoded by Kaposi's sarcoma-associated herpesvirus which resembles a spliced variant of human survivin. EMBO J 21: 2602-2615

Yamada Y, Kuroiwa T, Nakagawa T, Kajimoto Y, Dohi T, Azuma $\mathrm{H}$, Tsuji M, Kami K, Miyatake S (2003) Transcriptional expression of survivin and its splice variants in brain tumors in humans. J Neurosurg 99: $738-745$
You L, He B, Xu Z, Uematsu K, Mazieres J, Fujii N, Mikami I, Reguart N, McIntosh JK, Kashani-Sabet M, McCormick F, Jablons DM (2004a) An anti-Wnt-2 monoclonal antibody induces apoptosis in malignant melanoma cells and inhibits tumor growth. Cancer Res 64: 5385-5389

You L, He B, Xu Z, Uematsu K, Mazieres J, Mikami I, Reguart N, Moody TW, Kitajewski J, McCormick F, Jablons DM (2004b) Inhibition of Wnt2 -mediated signaling induces programmed cell death in non-small-cell lung cancer cells. Oncogene 23: 6170-6174

Zhang SQ, Qiang SY, Yang WB, Jiang JT, Ji ZZ (2004a) Expression of survivin in different stages of carcinogenesis and progression of breast cancer. Ai Zheng 23: 697-700

Zhang T, Fields JZ, Ehrlich SM, Boman BM (2004b) The chemopreventive agent sulindac attenuates expression of the antiapoptotic protein survivin in colorectal carcinoma cells. J Pharmacol Exp Ther 308: $434-437$

Zhang T, Otevrel T, Gao Z, Ehrlich SM, Fields JZ, Boman BM (2001) Evidence that APC regulates survivin expression: a possible mechanism contributing to the stem cell origin of colon cancer. Cancer Res 61: $8664-8667$

Zhang W, Hanks AN, Boucher K, Florell SR, Allen SM, Alexander A, Brash DE, Grossman D (2004c) UVB-induced apoptosis drives clonal expansion during skin tumor development. Carcinogenesis (Epub ahead of print)

Zhao H, Granberg F, Elfineh L, Pettersson U, Svensson C (2003) Strategic attack on host cell gene expression during adenovirus infection. J Virol 77: $11006-11015$

Zhu N, Gu L, Findley HW, Li F, Zhou M (2004) An alternatively spliced survivin variant is positively regulated by p53 and sensitizes leukemia cells to chemotherapy. Oncogene 23: 7545-7551

Zhu Y, Roshal M, Li F, Blackett J, Planelles V (2003) Upregulation of survivin by HIV-1 Vpr. Apoptosis 8: 71-79 